\title{
CIPHER, A POLARIMETER TELESCOPE CONCEPT FOR HARD X-RAY ASTRONOMY
}

\author{
R. M. CURADO DA SILVA ${ }^{1,2, *}$, E. CAROLI ${ }^{3}$, J. B. STEPHEN ${ }^{3}$ and P. SIFFERT ${ }^{1}$ \\ ${ }^{1}$ Laboratoire PHASE, CNRS, 23 rue do Loess, BP 20CR, 67037 Strasbourg CEDEX 2, France \\ ${ }^{2}$ Departamento de Física da Universidade de Coimbra, 3000 Coimbra, Portugal \\ ${ }^{3}$ IASF-Sezione di Bologna, Via Gobetti 101, 40129 Bologna, Italy \\ (*author for correspondence, e-mail: ruisilva@saturno.fis.uc.pt)
}

(Received 27 November 2003; accepted 18 February 2004)

\begin{abstract}
The polarisation of astrophysical source emission in the energy range from a few tens of keV up to the $\mathrm{MeV}$ region is an almost unexplored field of high-energy astrophysics. Till date, polarimetry in astrophysics-in the energy domain from hard X-rays up to soft $\gamma$-rays-has not been pursued due to the difficulties involved in obtaining sufficient sensitivity. Indeed for those few instruments that are capable of performing this type of measurement (e.g. the COMPTEL instrument on the Compton Gamma-ray Observatory and the IBIS instrument on INTEGRAL), polarimetry itself plays a secondary role in the mission objectives, as the efficiencies $(0.5 \%$ and $10 \%$ maximum, respectively) and polarimetric $Q$ factors ( 0.1 and 0.3 , respectively) are relatively limited. In order to perform efficient polarimetric measurements for hard X-ray and soft gamma-ray sources, with an instrument of relatively robust and simple design, a CdTe based telescope (CIPHER: Coded Imager and Polarimeter for High Energy Radiation) is under study. This instrument is based on a thick $(10 \mathrm{~mm})$ CdTe position-sensitive spectrometer comprising four modules of $32 \times 32$ individual pixels, each with a surface area of $2 \times 2 \mathrm{~mm}^{2}$ (about $160 \mathrm{~cm}^{2}$ total detection area). The polarimetric performance and design optimisation of the CIPHER detection surface have been studied by use of a Monte Carlo code. This detector, due to its intrinsic geometry, can allow efficient polarimetric measurements to be made between $100 \mathrm{keV}$ and $1 \mathrm{MeV}$. In order to predict the polarimetric performance and to optimise the design and concept of the CIPHER detection plane, a Monte Carlo code based on GEANT4 library modules was developed to simulate the detector behaviour under a polarised photon flux. The Compton double event efficiency, as well bi-dimensional double event distribution maps and the corresponding polarimetric modulation factor will be presented and discussed. Modulation $Q$ factors better than 0.50 and double event total efficiencies greater than $10 \%$ were calculated in the energy range between $100 \mathrm{keV}$ and $1 \mathrm{MeV}$. Herein we will present and discuss the general problems that affect polarimetric measurements in space, such as the inclination of the source with respect to the telescope optical axis and background radiation. $Q$ factor calculations for several beam inclinations as well as for background together with simulated astronomical sources will be presented and discussed.
\end{abstract}

Key words: astrophysics, flight instruments, Monte Carlo, polarimetry, semiconductor detectors

\section{Introduction}

Astrophysical sources of X-ray and $\gamma$-ray emission have been studied mostly through spectral and timing analyses. Other observational parameters can however, give additional information about constitution, physical processes and emissions

Experimental Astronomy 15: 45-65, 2003.

(C) 2004 Kluwer Academic Publishers. Printed in the Netherlands. 
of the various types of objects. Through polarimetric measurements we can obtain an extra observational parameter that allows, for example, the study of emission mechanisms that are associated with strong magnetic fields present in the source. These sources could be for example: pulsars, solar flares, active galactic nuclei, galactic black holes or even of unknown origin like those responsible for the production of Gamma-Ray Bursts. Till date, polarimetry in astrophysics, in the energy domain from hard X-rays up to soft $\gamma$-rays, is a field which has not been developed, in fact no dedicated polarimeters have ever been launched in space or on a balloon borne experiment, and only a few of these dedicated instruments have been proposed (Lei et al., 1997). Indeed for those few instruments that are capable of performing this type of measurement, polarimetry itself plays a secondary role in the mission objectives; the main purpose of these instruments being to perform spectroscopic imaging and timing analysis of celestial sources. This is the case for the COMPTEL (Schönfelder et al., 1993) (Imaging Compton Telescope) instrument on board CGRO (Compton Gamma-ray Observatory) and IBIS (Gehrels and Winkler, 1996) (Imager on Board the INTEGRAL Satellite) that is part of the INTEGRAL (INTErnational Gamma-Ray Astrophysics Laboratory) mission. The double event Compton efficiencies for these two instruments $(0.5 \%$ and $10 \%$ maximum, respectively) and their polarimetric $Q$ factors ( 0.1 and 0.3 , respectively) are not optimised on these instruments.

With the aim of building instruments with enhanced capabilities to perform polarimetric studies of hard X-ray and soft gamma ray sources, a collaboration has been made between the Laboratoire PHASE (PHysique et Applications des SEmi-conducteurs), CNRS, Strasbourg, France and the IASF (Istituto di Astrofisica Spaziale e Fisica Cosmica)—Sezione di Bologna, CNR, Italy. An instrument has been proposed for hard X-ray and soft gamma ray $(100 \mathrm{keV}-10 \mathrm{MeV})$ polarimetry in astrophysics, known as the CIPHER (Coded Imager and Polarimeter for High Energy Radiation) telescope (Caroli et al., 2000). The novel design of this telescope, using a $32 \times 32$ matrix of thick CdTe micro-spectrometers as the detection plane will allow this type of measurement to be performed for the first time by a dedicated instrument. The principle of operation is based on Compton polarimetry, particularly on the asymmetry of the double event (photons that first undergo a Compton interaction and are then absorbed through a second interaction with the material) distribution caused by a polarised beam. This detector, due to its intrinsic material properties and geometric shape promises efficient polarimetric measurements between $100 \mathrm{keV}$ and $1 \mathrm{MeV}$. The design and general characteristics of this instrument, which can represent a suitable option for future hard X-rays and soft gamma rays polarimetry stratospheric balloon flights or satellite missions, are described in the following section.

In order to optimise the design and the concept of the CIPHER telescope, we have developed a Monte Carlo code based on GEANT4 library modules to simulate the behaviour of the detector under various types of polarised photon beams, including the Crab pulsar as a reference astrophysical source, and under some of 
the main operating conditions associated with a balloon-borne experiment that is under development, such as the beam inclination and the influence of background on the performance of this instrument.

\section{Code implementation: Compton polarimetry and detection geometry}

GEANT4 library modules are a suitable tool to implement a Monte Carlo code that simulates the behaviour of a polarimetric device such as the CIPHER telescope detection plane. The code development was carried out mainly in two stages. First, the physical concepts associated with electromagnetic interactions and particularly the polarised Compton scattering and secondly the detection geometry concepts: the beam, the detection plane and read-out geometry.

\subsection{COMPTON SCATTERING POLARIMETER AND GEANT4 MODIFICATIONS ON G4POLARISEDCOMPTONSCATTERING CLASS}

CIPHER telescope polarimetric measurements are based on the fundamental concepts associated with polarised Compton interactions. In the considered energy range $(100 \mathrm{keV}$ to $1 \mathrm{MeV})$, this electromagnetic interaction can be the cause of a non-uniformity in the spatial distribution of the detected polarised photons. After going through Compton scattering, the polarised photons' new direction depends on the orientation of its polarisation vector before the interaction. If these polarised photons go through a new interaction inside the detector, the position information of the first and the second interaction (double event) allow us to infer the polarisation degree and direction of the radiation beam. The Klein-Nishina cross-section for linearly polarised photons gives us an azimuthal dependency for the scattered photons:

$$
\frac{d \sigma}{d \Omega}=\frac{r_{0}^{2}}{2}\left(\frac{E^{\prime}}{E}\right)^{2}\left[\frac{E^{\prime}}{E}+\frac{E}{E^{\prime}}-2 \sin ^{2} \theta \cos ^{2} \varphi\right]
$$

where $r_{0}$ is the classical electron radius, $E$ and $E^{\prime}$ are the energies of the incoming and outgoing photons respectively, $\theta$ the angle of the scattered photon and $\varphi$ is the angle between the scattering plane (defined by the incoming and outgoing photon directions) and incident polarisation plane (defined by the polarisation vector and the direction of the incoming photon). As can be seen from Equation (1), fixing all other parameters the probability varies with the azimuthal angle $\varphi$. The maximum relative difference between the cross-section values arises for $\varphi=0^{\circ}$ - when cross-section reaches a minimum-and $\varphi=90^{\circ}$, where the cross-section reaches a maximum. However this relative difference is maximised for an angle $\theta_{M}$, dependent on the incident photon energy. For hard $\mathrm{X}$-rays and soft gamma-rays the $\theta_{M}$ value is always around $90^{\circ}$ (Namito et al., 1993). The proposed CIPHER instrument is very suitable for polarimetric measurements since its planar configuration fits an essential 
condition for good polarimetric sensitivity: after a Compton interaction, outcoming photons with low and high $\theta$ values-when polarimetric asymmetries are less pronounced - take a detection plane divergent direction, while those emerging from interactions with $\theta$ close to $90^{\circ}$ have a stronger probability of going through a second interaction inside the detector. Thus with this rather simple design, we succeed in selecting the most interesting double events for the polarimetric source study.

Older versions of the GEANT4 (until 4.2.x versions) libraries possessed an unfinished class for polarised Compton scattering (G4PolarizedComptonScattering). An essential $\varphi$ generation routine as a function of a random number (Matt et al., 1996) was missing. Since $\varphi$ is given by an implicit expression, a dedicated routine was developed and included in the GEANT 4 code $\varphi$ that uses a simple numerical interpolation approach in order to generate $\varphi$ according to the distribution given by Equation (1). This correction is currently included in the latest versions of GEANT4 (4.3.x).

\subsection{CIPHER DETECTION IMPLEMENTED CODE: BEAM CHARACTERISTICS, DETECTION PLANE AND READOUT GEOMETRY}

Using the GEANT4 library modules, it was possible to construct an approximate model of the CIPHER position-sensitive detector, as well as the polarised beam characteristics that irradiates each detection plane module as shown in Figure 1. In Table I, the geometrical dimensions and material characteristics of the various parts of the detector are indicated as they were implemented in the Monte Carlo simulation code.

Each pixel is labelled by an identification number ( $x$ pixel ID, $y$ pixel ID) in the $x-y$ plane, containing the detector surface. The irradiation of the detector is simulated by a $100 \%$ linearly polarised beam along the $z$-axis uniformly covering the four central pixels: $(15,15),(15,16),(16,15)$ and $(16,16)$. Then for each of the surrounding pixels, we obtain the number of double and higher order events, as well as the respective energy deposition.

\section{Preliminary model: Polarimetric $Q$ factor and efficiencies}

The performance of a scattering polarimeter instrument can be evaluated by analysing the distribution of double events through the polarimetric modulation factor, $Q$. This is obtained by integrating the Compton polarimetric differential cross section formula over the solid angles defined by the physical geometry of the detection plane and can be calculated by means of

$$
Q=\frac{N_{y}-N_{x}}{N_{y}+N_{x}}
$$




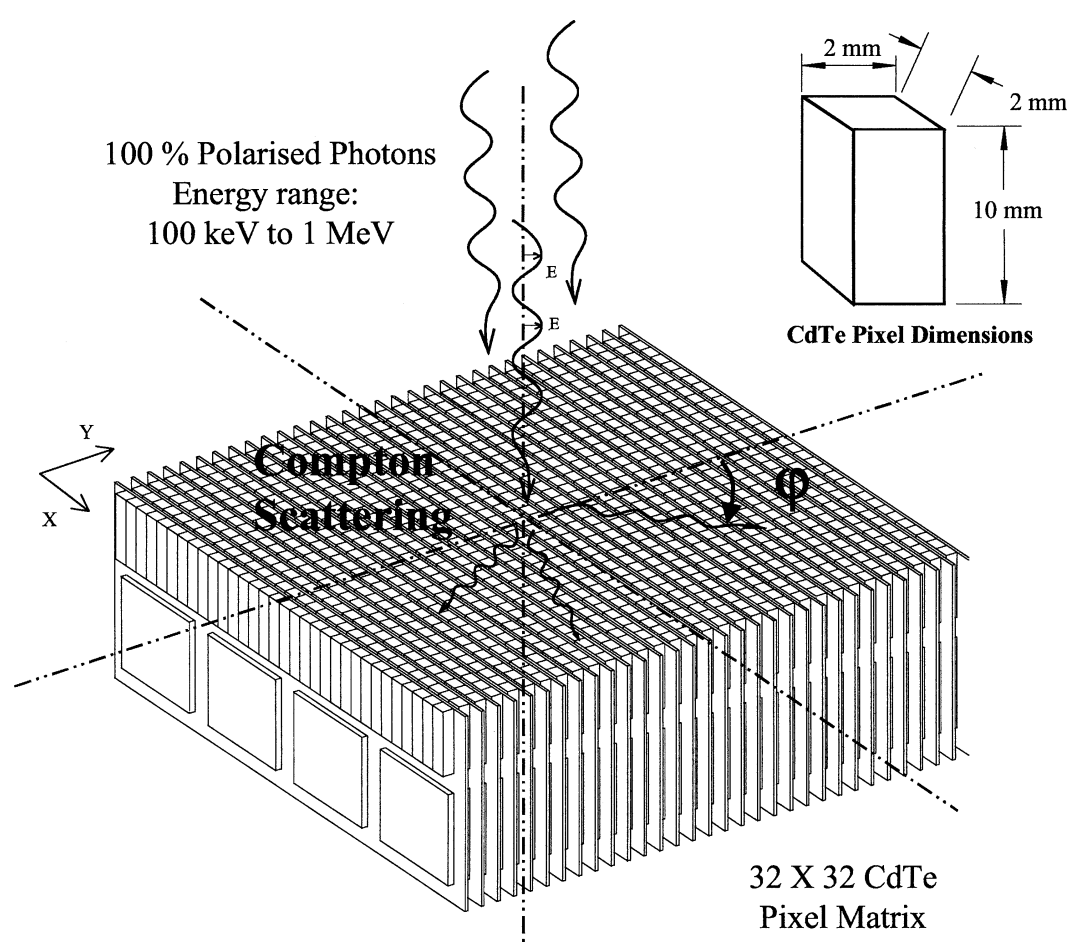

Figure 1. Schematic representation of a CIPHER polarimeter module and the respective pixel dimensions.

TABLE I

CIPHER polarimeter characteristics implemented in GEANT4 simulation code

\begin{tabular}{ll}
\hline Detector material & $\mathrm{CdTe}$ \\
Detector material density & $6.1 \mathrm{~g} / \mathrm{cm}^{3}$ \\
Active pixel size & $2 \times 2 \mathrm{~mm}^{2}$ \\
Pixel thickness & $10 \mathrm{~mm}$ \\
Linear module pixels pitch & $0.1 \mathrm{~mm}$ \\
Linear module pixels & $32 \mathrm{CdTe}$ micro-crystals \\
Linear module dimensions & $32 \times 0.1 \mathrm{~mm}^{2}$ \\
Linear module support material & $\mathrm{Al}_{2} \mathrm{O}_{3}$ \\
Linear module support density & $3.97 \mathrm{~g} / \mathrm{cm}^{3}$ \\
Linear module support thickness & $0.5 \mathrm{~mm}$ \\
Linear module pitch & $1.0 \mathrm{~mm}$ \\
Matrix module & $32 \times 32$ pixels \\
Number of modules & 4, one per quadrant \\
\hline
\end{tabular}




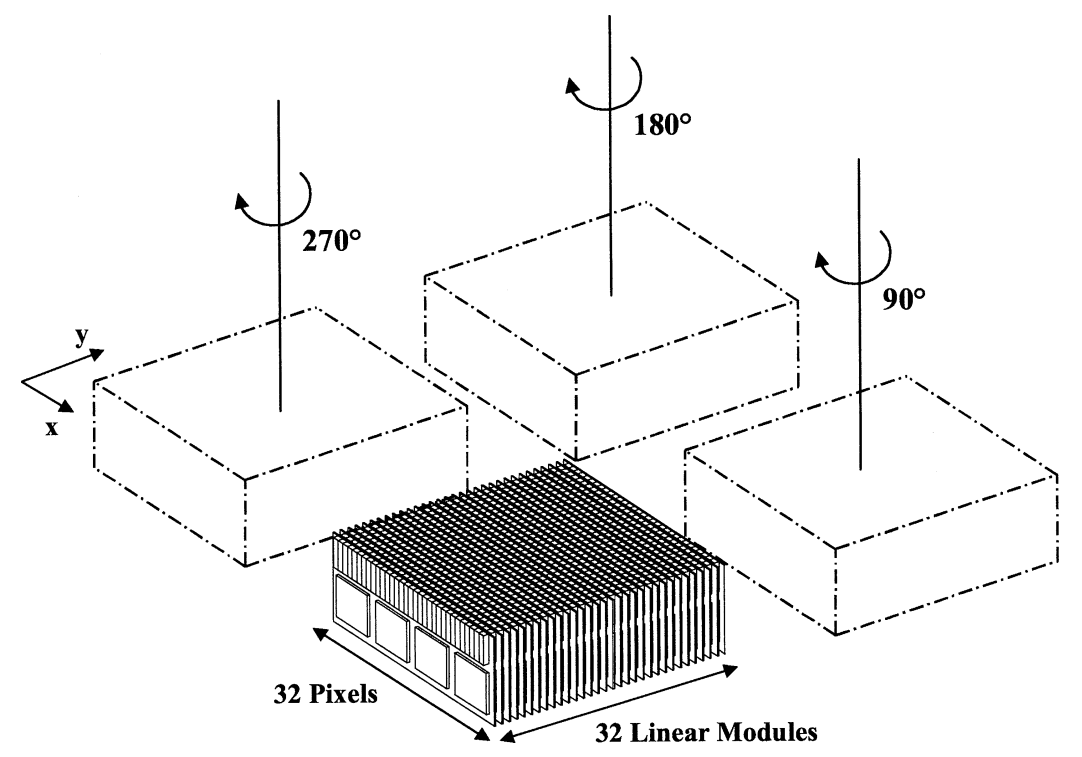

Figure 2. The four CIPHER modules spatial disposition in the detection plane. The rotation of each module is shown.

Equation 2 states that the $Q$ factor can be evaluated through the difference of counts in orthogonal directions over the detection plane, with respect to a polarised beam whose electric vector points in the $x$ direction. $N_{x}$ and $N_{y}$ are the number of counts in each of the orthogonal directions.

As can be seen in Table I, there is an asymmetry in pixel disposition in the orthogonal $x$ and $y$ directions of the detector plane. In each of the 32 linear modules the square $(2 \mathrm{~mm} \times 2 \mathrm{~mm})$ pixels are separated by $0.1 \mathrm{~mm}$, but each of these modules are $3 \mathrm{~mm}$ distant from adjacent modules and therefore in this direction the pixel pitch is $3 \mathrm{~mm}$ with a $1 \mathrm{~mm}$ gap. This technical constraint leads to an inherent detector asymmetry in the distribution of double events even if radiation is non-polarised, because the distance between two pixels that Compton photons have to cover is not the same in each of the orthogonal $x$ and $y$ directions. To correct this effect, we use the same four modules, but each one rotated $90^{\circ}$ in relation to adjacent modules (Figure 2). Double events obtained in each module are then summed in a unique $32 \times 32$ matrix, that takes into account the fact that the modules are rotated. In this way, we expect to cancel the inherent asymmetry due to different pixel pitch along the $x-y$ directions.

Results illustrating the double event distribution for each module, as well as the total distribution from the sum of the four modules into one single $32 \times 32$ matrix are shown in Figure 3. In this measurement, we irradiated each module with a $100 \%$ polarised $300 \mathrm{keV}$-beam covering uniformly each set of four central pixels as described above. As can be seen, the module rotation technique is very effective in cancelling the single module pixel pitch asymmetry effect, thereby 


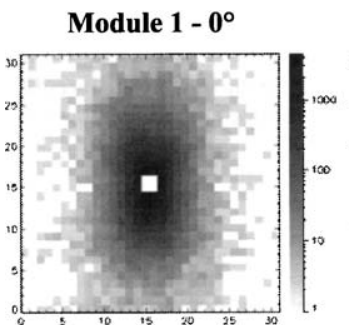

Module 3 - $180^{\circ}$

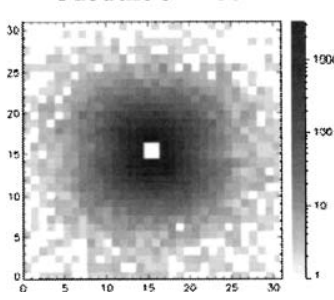

Module 2 - $90^{\circ}$

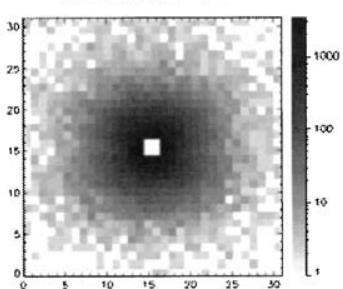

Module $4-270^{\circ}$

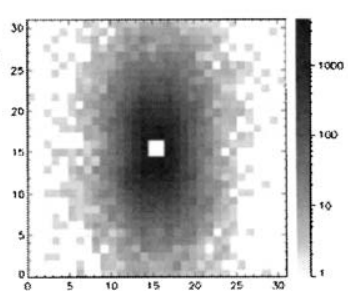

4 Modules $\Sigma$

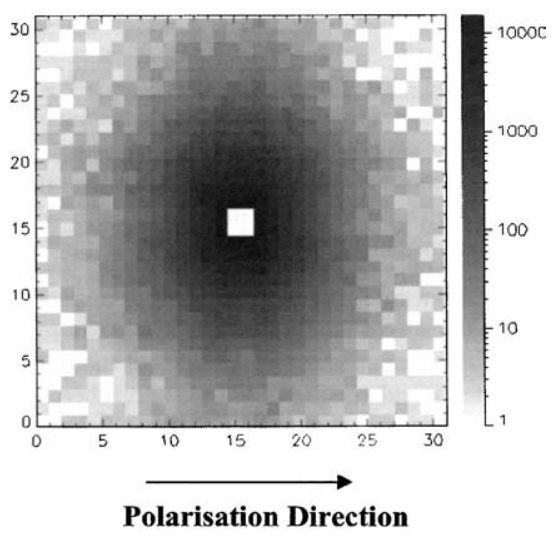

Figure 3. Four rotated module double event distributions for a $300 \mathrm{keV}$ full-polarised beam (in the $x$ direction) and respective sum in one single matrix of $32 \times 32$.

allowing an easier analysis of the polarisation information contained on the original beam.

In order to have a better idea of the effectiveness of this rotation technique, we also studied the case of one module with the same characteristics as the CIPHER modules except that the pixels pitch is uniform in both directions with a gap of $1 \mathrm{~mm}$. GEANT4 simulation maps obtained for a $500 \mathrm{keV}$ double event distribution produced by a $100 \%$ polarised beam are shown in Figure 4, together with a map showing the double scattered events distribution for an unpolarised beam. As can be seen, the direction orthogonal to the polarisation direction corresponds to maximum events detection, when the fully polarised beams are considered.

In all maps the central pixels $((15,15),(15,16),(16,15)$ and $(16,16))$ double events were ignored since they are directly irradiated and we want to study the distribution of double events around these central pixels.

A systematic study of the detector polarimetric performance was performed covering the energy range between $100 \mathrm{keV}$ and $1 \mathrm{MeV}$ in steps of $100 \mathrm{keV}$. The $Q$ factor was calculated for double events distributions originating from $5 \times$ $10^{6}$ photons of $100 \%$ linearly polarised beams. In Figure 5, we present the obtained polarimetric $Q$ factor excluding double events in the central pixels, first order (pixels adjacent to the irradiated central pixels) and second order pixels (those surrounding first order distance pixels). Comparison with polarimetric modulation factors obtained to the referred symmetric module is also shown, proving the reliability of our rotation method correction.

As demonstrated by the results shown in Figure 5, the $Q$ factor improves when we discard higher order distance pixel events. For instance, in the range from 
One symmetric module

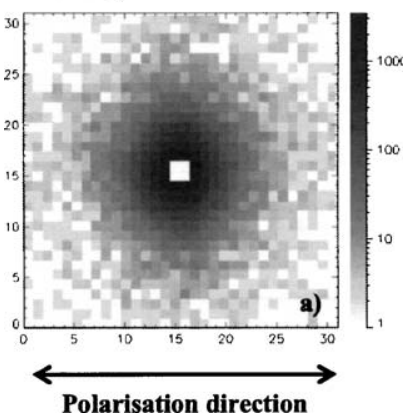

Four CIPHER modules

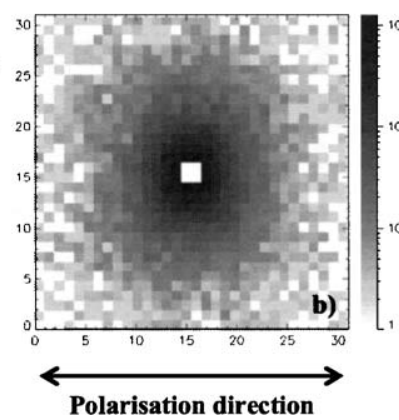

Four CIPHER modules

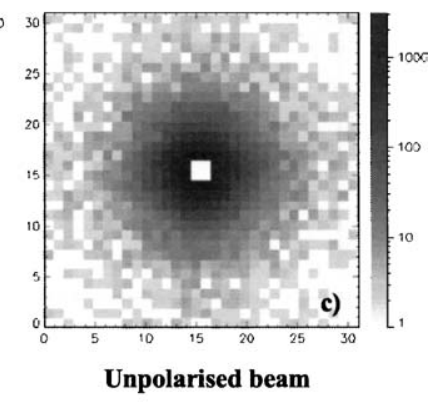

Figure 4. Maps comparing $500 \mathrm{keV}$ beam double events distribution in three cases: a) a $100 \%$ polarised beam (polarisation direction is shown) irradiating a symmetric type CIPHER module ( $1 \mathrm{~mm}$ pixel gap both in $x$ and $y$ directions), b) a $100 \%$ polarised beam irradiating four CIPHER rotated modules, and c) an unpolarised beam irradiating the same four CIPHER modules.

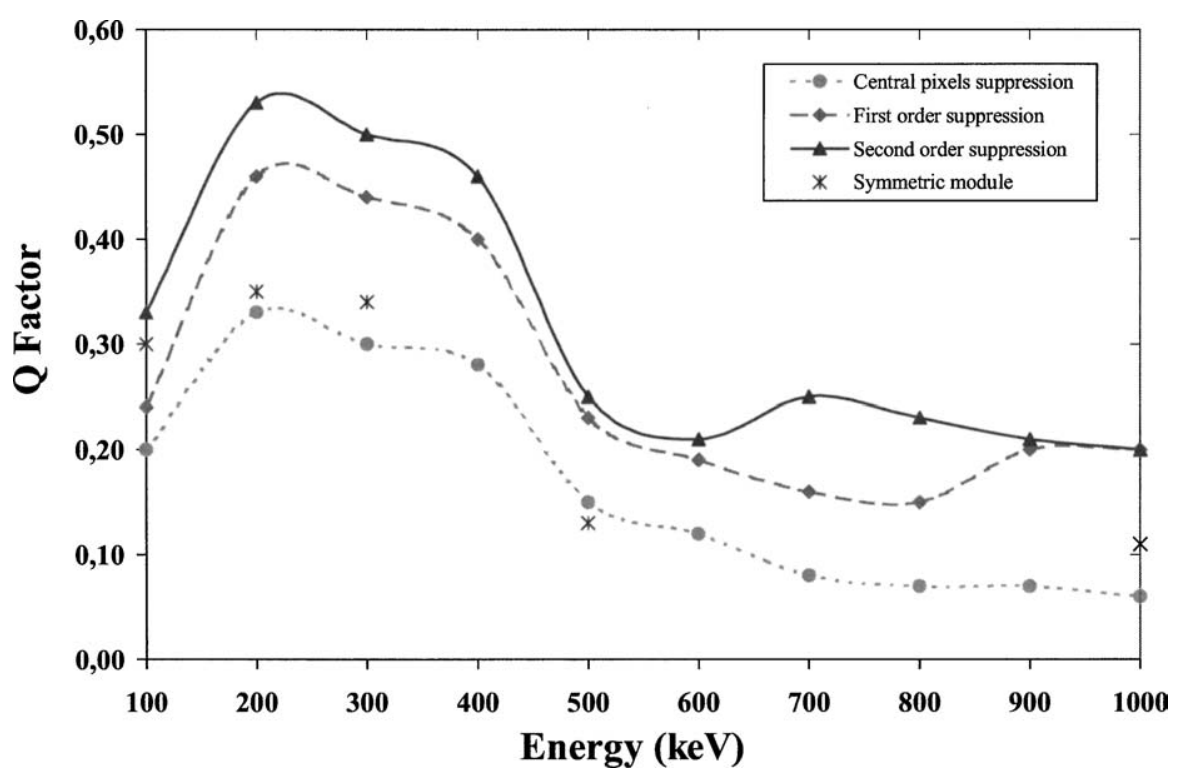

Figure 5. $Q$ Factor in function of energy for CIPHER telescope in the following cases: excluding only central pixel double events, excluding additional first order pixels double events, and additionally second order pixels double events.

200-400 keV, if we exclude all double events occurring inside central, fist order and second order pixels we can increase $Q$ factor from about 0.3 to a $Q$ of about 0.5 . This is due to the fact that we have a better definition of the angular distribution of scattered with respect to closer distance order from the irradiated pixels, since 


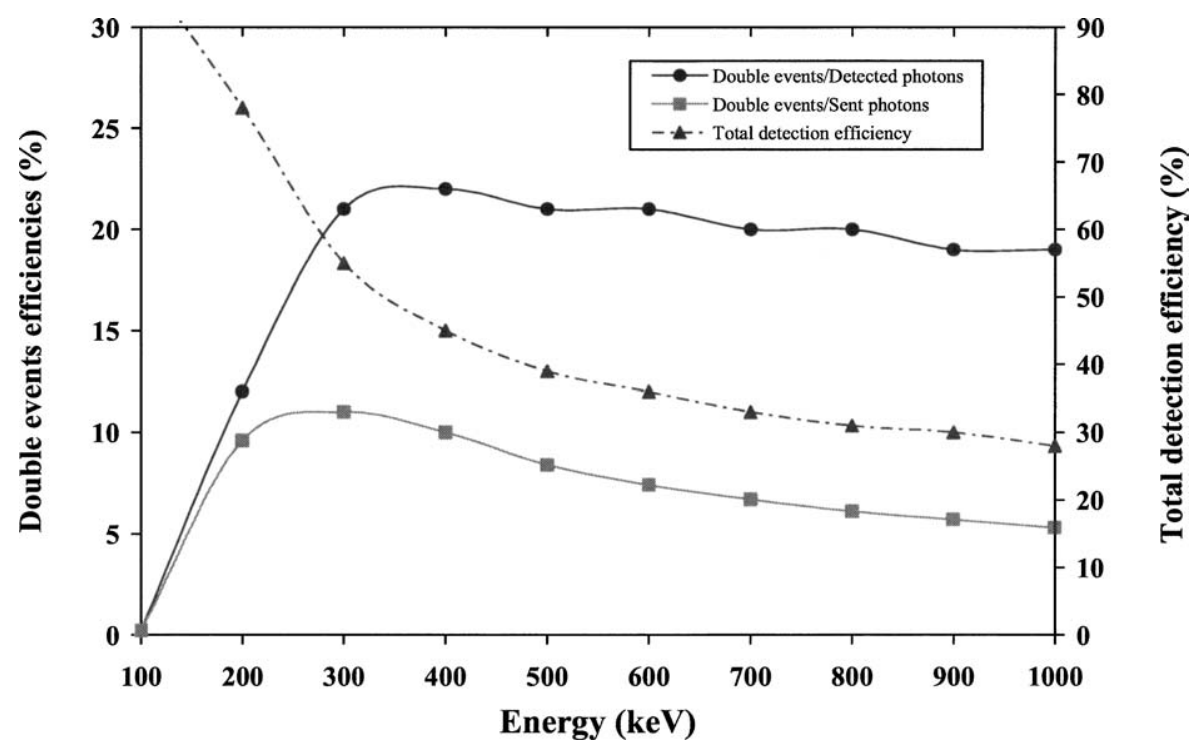

Figure 6. Efficiencies obtained for the CIPHER telescope in the energy range from $100 \mathrm{keV}$ to $1 \mathrm{MeV}$.

increasing the order of pixels that contribute to the double event statistics surrounding the detection plane centre, we are also increasing the average pixels number per order through which the distribution is going to spread, therefore leading to a better polarimetric figure definition. However, when we do this procedure, although we are improving our polarisation sensitivity, but we are also decreasing our global double event counts, thus reducing the efficiency of the device. Therefore depending on the astrophysical radiation source that we intend to study we must find the optimum product between $Q$ factor and efficiency by integrating over the energy range previously defined (Caroli et al., 2000). If the radiation source is very intense, which means that efficiency is less important, it is a better option to exclude lower order pixels in order to improve our sensibility. On the other hand, if the radiation source is very faint all pixels must be taken into account in order to maximize the efficiency of our instrument.

As described, since efficiency is an important factor when analysing distant and very often faint astronomical objects, we present efficiencies obtained for 100 to $1000 \mathrm{keV}$ energy range in Figure 6. Double events relative (double events/total detected photons) and absolute efficiency (double events/total sent photons over detector plane) show very poor efficiency for low energies $(\sim 100 \mathrm{keV})$, a rapid increase until about $300 \mathrm{keV}$, and then followed by a smooth decrease until $1 \mathrm{MeV}$. Total efficiency (all order events/total sent photons) show very high values for low energies-where the main contribution is due to single events-decreasing until $1 \mathrm{MeV}$, but even at this energy more than a quarter of events are detected. 


\section{In flight distributions}

\subsection{FULL SURFACE IRRADIATION AND CRAB SPECTRA}

In chapter 3, we made a preliminary approach to CIPHER polarimetric capabilities, where we had presented a simple study in order to evaluate the polarimetric performance limits. As we described, the results were obtained for a beam configuration where only four central pixels were irradiated. However, in a future scientific CIPHER mission the full detection surface of the four CdTe matrix modules will be exposed to incoming high energy radiation. In order to get closer approximation to the in-flight measurements conditions we changed our simulation model by irradiating the entire detector plane. Also a beam where the energy distribution is based on Crab nebula like power law $\left(\propto \mathrm{E}^{-2}\right.$ photons $\left./\left(\mathrm{cm}^{2} \cdot \mathrm{s} \cdot \mathrm{keV}\right)\right)$ was simulated, allowing us to approximate an event distribution closer to that produced by flux from a real astrophysical object.

Although in actual in-flight measurements, temporal order of the events hits is almost impossible to know both the intrinsic time response of CdTe detectors and insufficient speed of the read-out electronics, when simulating this condition with the GEANT4 libraries, interaction order is always a known parameter. Actually, each photon trajectory is followed since it is generated until it is either absorbed by the detector or it goes out of the simulation overall volume, and so the interaction order is thus always known for each photon. However, even if the event order is not known when analysing Compton telescope data, polarimetric measurement can be performed. In fact, which interaction is taken to be first is unimportant due to the inherent $90^{\circ}$ double events distribution symmetry (Lei et al., 1997).

Making use of the GEANT4 tools, we simulated the irradiation of the full CIPHER detection plane with a monochromatic beam, where for each photon was recorded the following data: number of event, multiplicity of the interaction (number of hits), and for each hit the pixel ID and the energy deposited. As expected, irradiating the entire detector surface no trivial double event distribution arises when we observe the respective map, as we did in the former chapter. However, we present a method to analyse this kind of distribution where each pixel is considered, like if it was a central pixel of a matrix of pixels. Actually, the recorded photon history tells us which pixel goes under a first Compton interaction, which goes under a second interaction and other order interactions if it is the case. Picking one pixel at the time we are going to place it at the centre of a matrix of pixels and for each time it went under a first order interaction, we sum the energy deposited in the pixels at the same relative distance as its real position. Proceeding this way, for each pixel we have a distribution of energy deposition as if this pixel was a central pixel of a matrix. Summing all pixels double order event distributions like they were all the same central pixel we obtain a total double events distribution as if we had irradiated only a central pixel with the same number of photons that we used to irradiate all detector surface. At the same time, this total distribution is 
TABLE II

Polarimetric modulation $Q$ factor for full surface CIPHER telescope irradiation

\begin{tabular}{ll}
\hline Energy $(\mathrm{keV})$ & $Q$ factor \\
\hline 200 & 0.36 \\
300 & 0.33 \\
500 & 0.28 \\
1000 & 0.20 \\
Crab & 0.33 \\
\hline
\end{tabular}
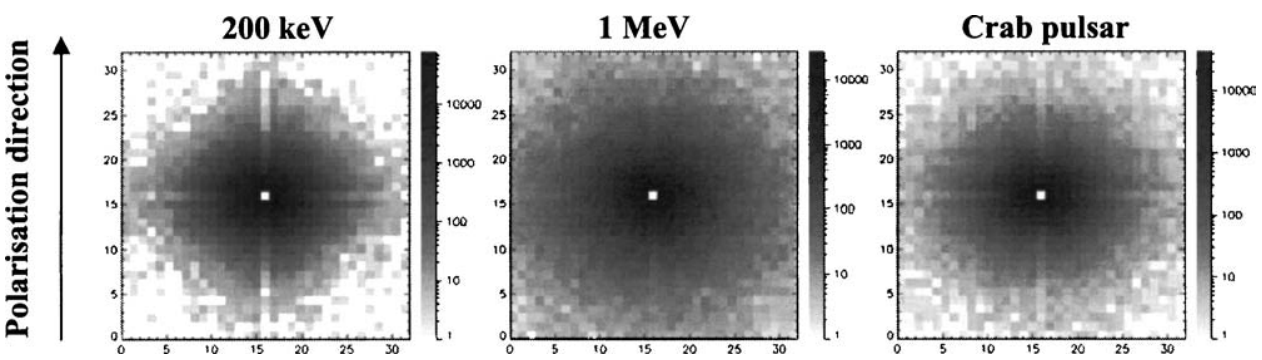

Figure 7. Double interaction event maps obtained for full surface detector irradiation after applying the central pixel method to each pixel of CIPHER telescope four modules. The represented distributions correspond to irradiation with monoenergetic beams of $200 \mathrm{keV}$ and $1 \mathrm{MeV}$ and with a Crab power law spectrum. Beams were $100 \%$ polarised according to the represented direction.

self-corrected as the edge pixel asymmetric distributions cancel each other when they are added together. This method allows us to maximise the use of detector surface area (each pixel is a scattering centre and a scattered photon detector) and at the same time to keep the polarimetric results comparable with the case already studied in chapter 3.

In Table II we present the modulation $Q$ factor values obtained for the full detector plane irradiation for a $100 \%$ linearly polarised photon beam, where all order pixels we considered in our calculations.

As can be seen results are very similar to those obtained when we irradiate only four central pixels per each module (Figure 5). We get even a slight improvement of $Q$ factor due to double events distribution around a single pixel—four pixels in the previous study-increasing this way the distribution figure resolution.

In Figure 7 we show the double events distribution obtained after the described method has been applied to all four CIPHER modules CdTe pixels.

Modulation $Q$ factor results as well as a double interaction event map are also presented for the power law Crab distribution (Table II and Figure 7). Because this distribution is more concentrated at lower energies, $Q$ factor and distribution map are very similar to those obtained around the range between 200 and $300 \mathrm{keV}$. 
Herein, for simplicity, we present double events distributions that are generated by linearly polarised beams whose polarisation vector is parallel to one of the principal axis of the detector $x$ or $y$. However during a balloon born or satellite experiment polarisation vector can have any orientation. Therefore several methods were developed in order to allow the analysis of double events distributions even if initially we do not know the polarisation vector direction of the incoming beam. One of these methods is the Moving Mask Technique (Swinyard et al., 1991). Through this method we are able to determine the direction at which the $Q$ factor reaches a maximum and therefore we are able to determine the polarisation vector of the incoming beam and the effective $Q$ factor associated to the double events distribution. For a high pixelisation level detection plane, as the CIPHER polarimeter, this method has negligible influence in the results obtained.

\subsection{INCLINED BEAM EFFECTS}

During the flight of a balloon borne experiment, several external factors can affect significantly the polarimetric measurements. One of the most important factors is the direction of the incoming photons from the polarised radiation source with respect to the surface of the polarimeter. Previous studies (Curado da Silva et al., 2002) have always assumed that beams were orthogonal with respect to the detection plane. Therefore we present a study of CIPHER's polarimetric response as a function of the direction of a full-polarised beam. Following the model described in the previous section, we irradiated the four central pixels of the detector modules using both unpolarised and $100 \%$ polarised beams forming different inclination angles with the optical detector axis $z: 0^{\circ}, 2^{\circ}, 5^{\circ}$ and $10^{\circ}$ (Figure 8). Furthermore we implemented beams with different polarisation vector directions: along the $x$ - and $y$-axis.

As we can see in Figure 9, the beam inclination generates an asymmetric double event distribution around the irradiated pixels that has no relation with the beam polarisation. For an unpolarised beam at $2^{\circ}$ a component added to the distribution that was caused by beam inclination is visible. Due to the beam inclination some of the photons irradiating the central pixels surface can cross these pixels without interacting with the material and penetrate adjacent pixels to the central ones (first order pixels) and undergo a first interaction therein (Figure 8). For a polarised beam along the $y$ direction, the double event distribution is characterised by higher counts in the orthogonal direction (along the $x$ direction). Therefore the two contributions are added and the distribution is artificially more asymmetric. Obviously, when the beam polarisation is in the $x$ direction the two contributions are opposite and partially cancel each other.

The consequences of beam inclination on the polarimetric performance of the instrument are better understood when the respective $Q$ factors in an energy range between $100 \mathrm{keV}$ and $1 \mathrm{MeV}$ are calculated (Figures 10,11). We can see that an 


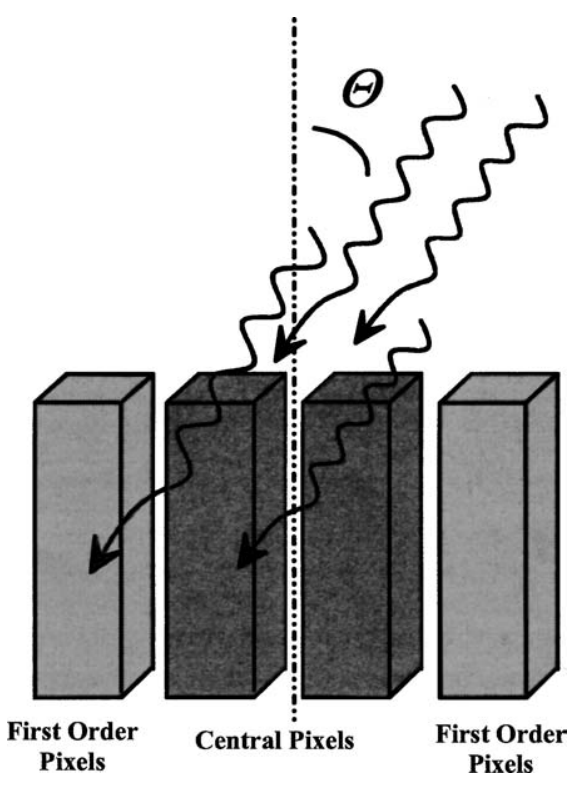

Figure 8. Schematic view of central pixels irradiation by a polarised inclined beam at angle $\Theta$ with respect to the detector optical axis.

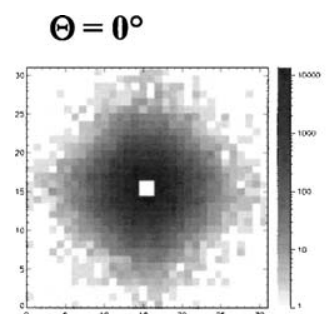

Unpolarised

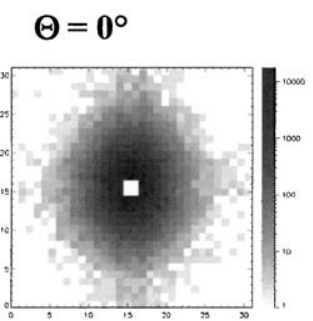

$100 \%$ polarised

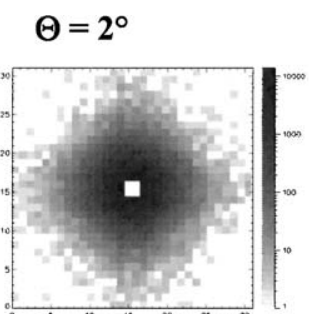

Unpolarised

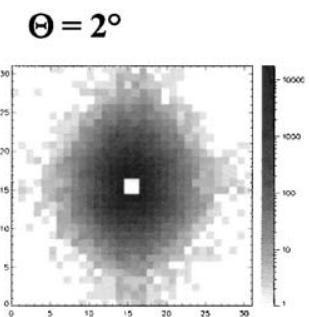

$100 \%$ polarised

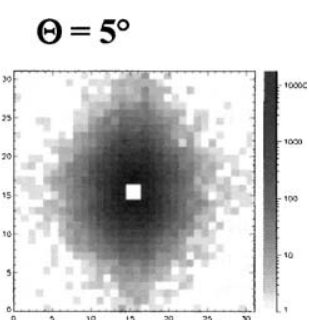

$100 \%$ polarised

$$
\Theta=10^{\circ}
$$

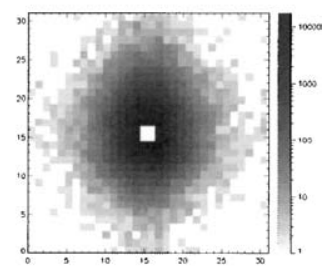

$100 \%$ polarised

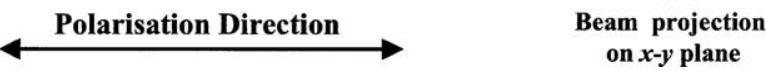

Figure 9. Double event distributions obtained with both unpolarised and $100 \%$ polarised monochromatic beam of $200 \mathrm{keV}$ irradiating the CIPHER telescope modules at various inclination angles $\Theta$ with respect to the telescope optical axis. 


\section{$0^{\circ}$ and $2^{\circ}$ inclined beams}

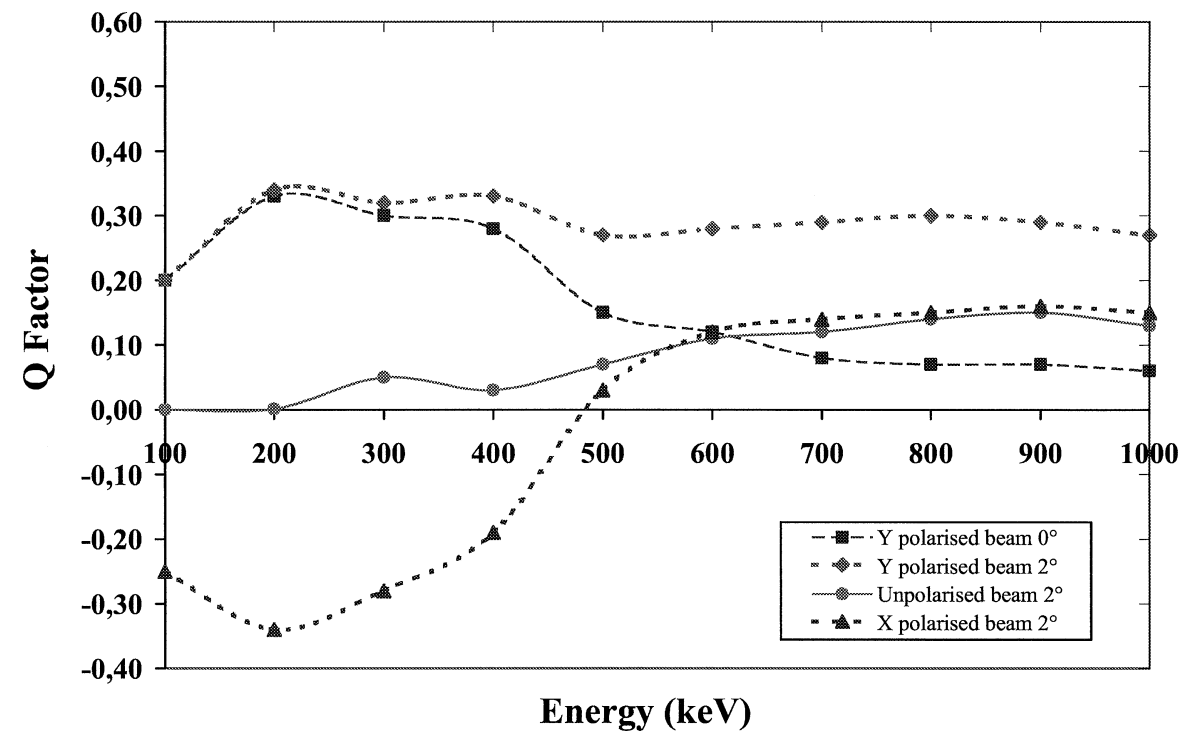

Figure 10. Evolution of $Q$ factor with energy for various types of beam: $100 \%$ polarised along $y$ inclined at $0^{\circ}, 100 \%$ polarised inclined along $y$ at $2^{\circ}$, unpolarised inclined at $2^{\circ}$ and $100 \%$ polarised along $x$ inclined at $2^{\circ}$.

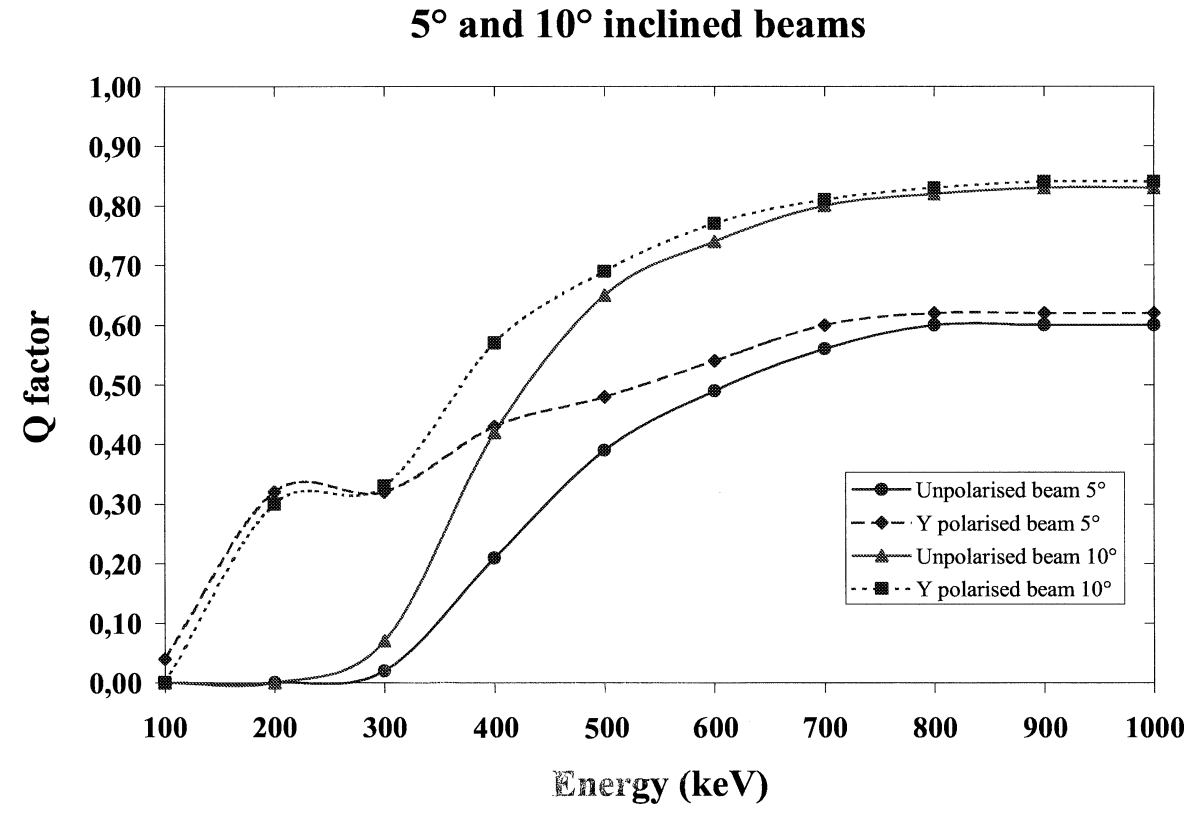

Figure 11. Evolution of $Q$ factor with energy for various types of beams: $100 \%$ polarised along $y$ inclined at $5^{\circ}$, unpolarised inclined at $5^{\circ}, 100 \%$ polarised along $y$ inclined at $10^{\circ}$ and unpolarised inclined at $10^{\circ}$. 
unpolarised beam at $2^{\circ}$ can artificially show a certain degree of polarisation, which increases with energy, above a few hundreds of $\mathrm{keV}$, when the $Q$ factor in an unpolarised beam should be always zero. Comparing the $Q$ factor evolution with energy for $100 \%$ polarised beams at $0^{\circ}$ (orthogonal beam) and $2^{\circ}$, we can deduce that the evolution of $Q$ with the energy of the beam is roughly the superposition of the curve corresponding to an unpolarised beam at $2^{\circ}$ plus the standard $Q$ factor curve for a $100 \%$ polarised beam of an orthogonal beam $\left(0^{\circ}\right)$. This shows clearly that contributions of beam polarisation and inclination on double event distributions increases the $Q$ factor artificially. The opposite is observed when the polarisation is along the $x$-axis. As we can see, the $Q$ factor (negative because we still use Equation (2)) after $300 \mathrm{keV}$ becomes lower, crossing zero at about $500 \mathrm{keV}$. This means that component due to beam inclination is more important than the polarisation component for the double event distribution above $500 \mathrm{keV}$. For higher beam inclination angles these effects are more pronounced and the polarimetric capabilities can be dramatically affected and an appropriated model shall be applied to recover the information about the polarisation degree of the incoming radiation.

This study shows the importance of the pointing system accuracy for an instrument designed for polarimetry. For the CIPHER telescope the foreseen pointing precision is lower than $1^{\circ}$. This precision should be sufficient so that double event distributions can be read directly with no need of correction methods.

\subsection{BACKGROUND NOISE EFFECTS ON POLARIMETRY}

Another factor that can be critical to polarimetric measurements is the background noise level caused both by cosmic radiation and indirect events caused by particles generated by photons cosmic rays interacting with the material of the elements that compose the telescope.

\subsubsection{Polarimetric $Q$ factor for a Crab like source superimposed to background distributions}

In our study we considered three types of background levels that were measured by two different instruments on stratospheric balloon borne experiments. These instruments are the PoRTIA (Piggyback Room Temperature Instrument for Astronomy) (Parsons et al., 1994) on board the GRIS (Gamma-Ray Imaging Spectrometer) mission that flew in Alice Springs and HEXIS (High Energy X-ray Imaging Spectrometer) (Slavis et al., 2000) that flew in Palestine, Texas, USA. Both are based on CdZnTe detection elements. PoRTIA houses a CdZnTe detector of $25 \times 25$ $\times 1.9 \mathrm{~mm}^{3}$ and on board the HEXIS instrument is included a CdZnTe detection plane of $11 \times 11 \times 2 \mathrm{~mm}^{3}$. As this CdZnTe material has very similar properties to CdTe, the crystals used on the detection plane of the CIPHER telescope, these backgrounds are very suitable for our study.

We used the background rates measured by two different configurations of the HEXIS CdZnTe detector shielding. In one configuration a full active shielding was 


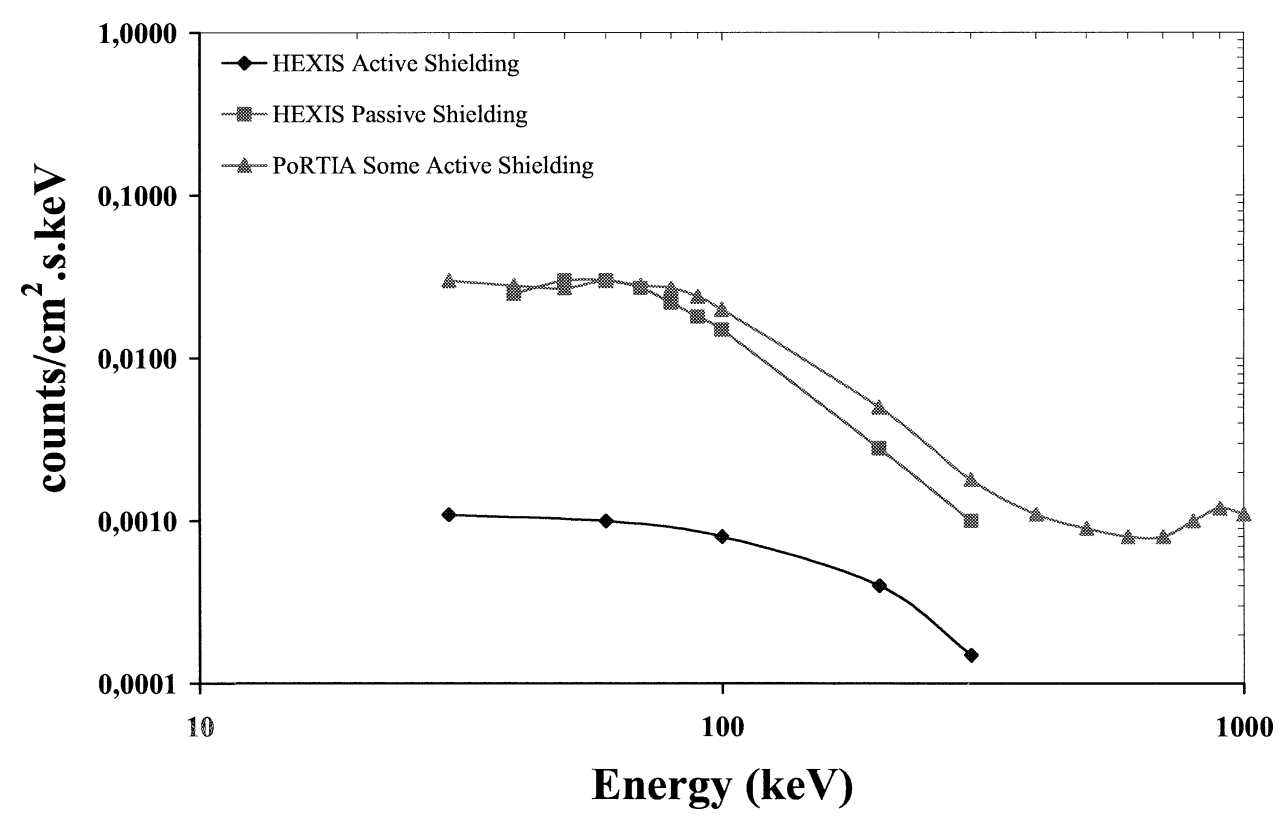

Figure 12. Background rates measured by HEXIS and PoRTIA instruments.

obtained by use of a $\mathrm{NaI}$ collimator and rear CsI elements producing a field of view of $20^{\circ}$. In a second configuration a partial active shielding was limited to the detection plane rear active shielding consisting of CsI elements so as to give a $140^{\circ}$ field of view. The published PoRTIA background rates correspond to measurements in a partial active shielding configuration for a $10^{\circ} \times 10^{\circ}$ field of view. The foreseen field of view for the CIPHER telescope is of about $10^{\circ}$, therefore the configurations that were chosen approach the CIPHER characteristics, except for the HEXIS $140^{\circ}$ configuration that was included on our study only in order to evaluate the difference between a full and a partial active shielding measured by the same instrument. The background rates measured by this instrument are shown in Figure 12. The data from the Alice Springs flights were divided by 0.72 to correct the difference in the geomagnetic latitudes between Palestine and Alice Springs (Curado da Silva et al., 2002).

A code was then implemented where four central pixels of the CIPHER modules were irradiated by a beam whose flux was obtained from the Crab nebula emission rate $\left(10 . \mathrm{E}^{-2}\right.$ photons $\left./\left(\mathrm{cm}^{2} \cdot \mathrm{s} \cdot \mathrm{keV}\right)\right)$ superposed on the three background rates measured by the HEXIS and PoRTIA instruments. The background rates shown in Figure 12 were fitted by an exponential function of the type: $y=C e^{-D x}$, where $C$ and $D$ are two constants. Furthermore, we divided the CIPHER energy operational interval (100 keV-1 MeV) into three bands: $100-300 \mathrm{keV}, 300$ $500 \mathrm{keV}$ and $500 \mathrm{keV}-1 \mathrm{MeV}$. The double event distributions obtained from these combinations of a $100 \%$ Crab polarised emission and the different unpolarised 


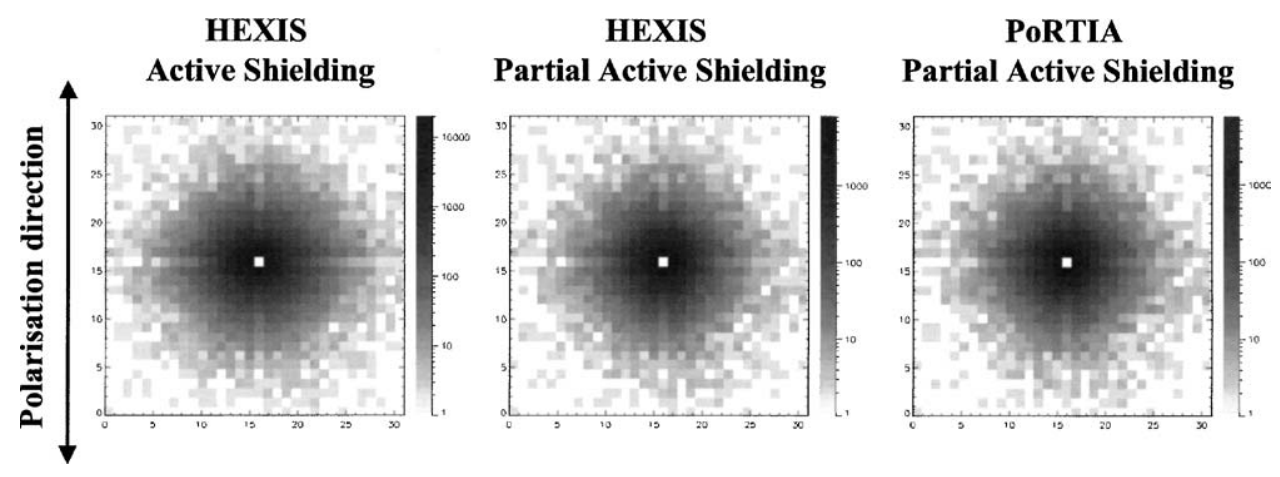

Figure 13. Double events distributions obtained through the superposition of Crab emission and several background measured rates in the band from 100 to $300 \mathrm{keV}$.

TABLE III

CIPHER $Q$ factors for combined Crab emission flux with measured radiation background counts in the range from $100 \mathrm{keV}$ to $1 \mathrm{MeV}$

\begin{tabular}{llll}
\hline & \multicolumn{3}{c}{ Modulation $Q$ Factor } \\
\cline { 2 - 4 } Flux type & 100 to $300 \mathrm{keV}$ & 300 to $500 \mathrm{keV}$ & $500 \mathrm{keV}$ to $1 \mathrm{MeV}$ \\
\hline $\begin{array}{l}\text { Crab + Background } \\
\quad \text { HEXIS Active Shielding) }\end{array}$ & 0.23 & 0.12 & 0.040 \\
$\begin{array}{l}\text { Crab + Background } \\
\quad \text { (HEXIS passive shielding) }\end{array}$ & 0.037 & 0.019 & 0.0060 \\
$\begin{array}{l}\text { Crab + Background } \\
\quad \text { PoRTIA partial active shielding) }\end{array}$ & 0.024 & 0.012 & 0.0029 \\
\hline
\end{tabular}

background measured fluxes (Figure 13) allow us to calculate the polarimetric $Q$ factor (Table III).

From the results shown in Table III we can see that the polarimetric performance is particularly affected by background radiation for higher energies, although we can observe that also the shielding configuration is a decisive factor for polarimetry. In fact, full active shielding is much more efficient than the partial shielding configurations. Fully active shielding allows polarimetric measurements of a celestial source such as the Crab with minimal degradation of the $Q$ factor values ( $Q$ is 0.31 in the full energy range from a Crab emission with no background). In the partial shielding configurations, the high background rates are responsible to decrease the $Q$ factors at least six times with respect to the no background conditions.

The CIPHER telescope foresees the use of shielding composed of a lateral tungsten passive shielding ( $>90 \%$ opaque at $100 \mathrm{keV}$ and a CsI(Tl) full active shielding ( $>70 \%$ opaque at $500 \mathrm{keV}$ ) for a $8^{\circ} \times 10^{\circ}$ field of view. Therefore we expect that polarimetric performances of our instrument will be similar to those obtained in the 


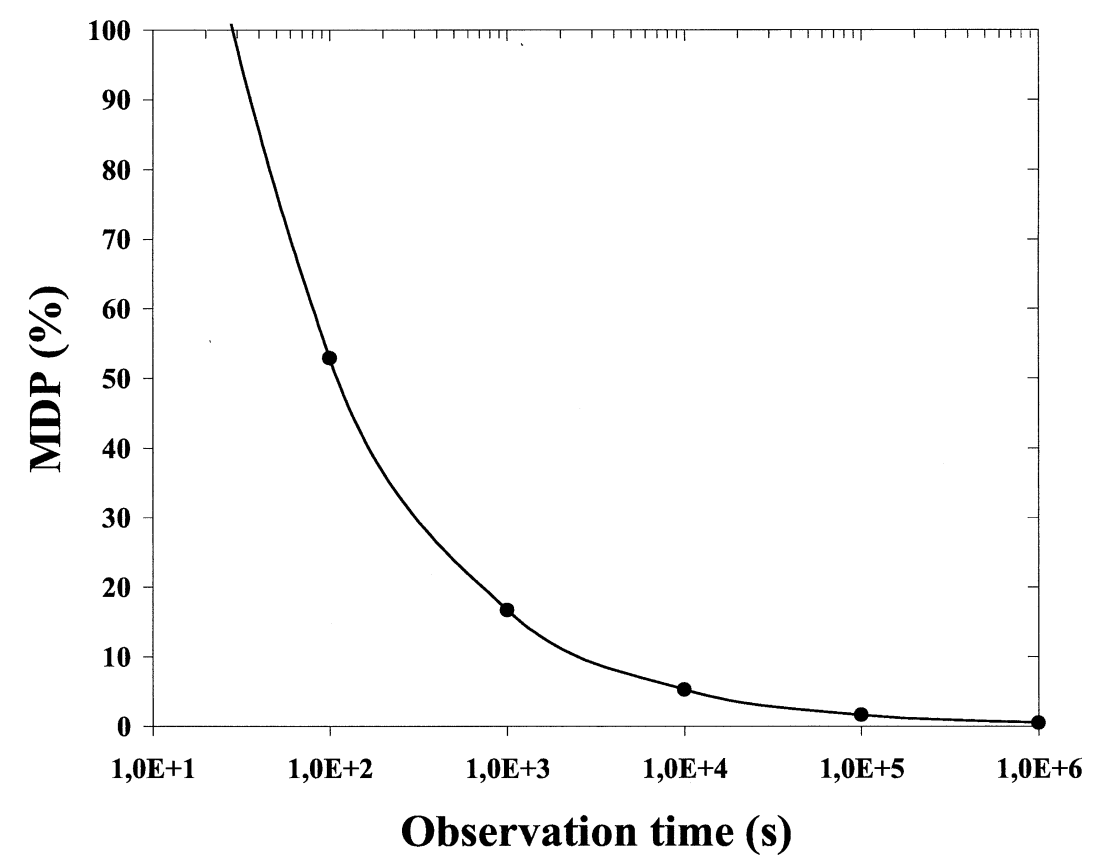

Figure 14. The MDP calculated for the CIPHER telescope when irradiated by a Crab nebula emission flux in presence of a background noise rate measured by HEXIS instrument (full active shielding configuration).

case of background levels measured by HEXIS in full active shielding configuration and only a small degradation of the $Q$ factor values will be observed.

\subsubsection{Minimum detectable polarisation (MDP) for a Crab distribution}

The minimum detectable polarisation of a polarimeter in the presence of background noise can be expressed by:

$$
\operatorname{MDP}(100 \%)=\frac{n_{\sigma}}{A \cdot \varepsilon \cdot S_{F} \cdot Q_{100}} \sqrt{\frac{A \cdot \varepsilon \cdot S_{F}+B}{T}}
$$

where $n_{\sigma}$ is the significance, $Q_{100}$ the modulation factor for a $100 \%$ polarised source, $\varepsilon$ the double detection efficiency, $A$ the polarimeter detection area in $\mathrm{cm}^{2}$, $S_{f}$ the source flux (photons $\cdot \mathrm{s}^{-1} \cdot \mathrm{cm}^{-2}$ ), $B$ is the double background rate (counts/s) and $T$ the observation time in seconds.

For a $3 \sigma$ detection significance, we calculated the MDP expected for the CIPHER instrument for the Crab emission in the presence of a background level corresponding to that measured by HEXIS in the full active shielding configuration (Figure 14). The CIPHER total active surface is about $160 \mathrm{~cm}^{2}$, the $Q_{100}$ factor for a double events distribution generated by a $100 \%$ polarised Crab emission in the 
energy range from $100 \mathrm{keV}$ to $1 \mathrm{MeV}$ is 0.31 for an overall double event efficiency of $6 \%$.

The MDP evolution with the observation time shows that the CIPHER instrument is potentially capable of measuring the Crab emissions polarisation to levels lower than $20 \%$ for an observation time of $10^{3} \mathrm{~s}$, about $5 \%$ for $10^{4} \mathrm{~s}$ and lower than $1 \%$ for $10^{6} \mathrm{~s}$ of observation time (observations longer than $10^{4} \mathrm{~s}$ were considered in order to compare with satellite observation times). These results obtained for the CIPHER telescope full operational energy range (100 keV to $1 \mathrm{MeV})$ indicate polarimetric performances better then those expected for the Crab emission from non-dedicated instruments such as INTEGRAL (MDP of $2 \%$ for an observation time of $10^{6} \mathrm{~s}$ without background), COMPTEL ( $30 \%$ for an observation time of $10^{6} \mathrm{~s}$ without background) or polarimeter projects like GIPSI (Kroeger et al., 1996) ( $1.4 \%$ for an observation time of $10^{6} \mathrm{~s}$ without background) and Lxe-TPC (Aprile et al., 1994) (3\% for an observation time of $10^{6} \mathrm{~s}$ without background).

\section{Conclusions}

Throughout this work we show that the instrument proposed for hard X-ray and soft $\gamma$-ray polarimetry is an interesting option for development, as potentially the CIPHER telescope can attain $Q$ factor values higher than 0.5 for energies between 200 and $400 \mathrm{keV}$, while in addition to this, it offers a large surface active area $\left(\sim 164 \mathrm{~cm}^{2}\right)$, a good efficiency in range due to the high cross section of the CdTe pixel material and the possibility of working simultaneously as an imager. Other instruments that cover a considerable part of CIPHER energy range like GIPSI polarimeter-based on germanium strips-can attain a $Q$ factor of 0.7 (Kroeger et al., 1996), but its energy range is limited to the interval between 70 and $300 \mathrm{keV}$, its efficiency is also limited to a maximum of 5\% and germanium demands always a complex and heavy cooling system. INTEGRAL however has its energy range between $200 \mathrm{keV}$ and $3 \mathrm{MeV}$, but $Q$ factor is always less than 0.3 (Stephen et al., 2001). We have also shown the validity of module rotation technique to correct asymmetry in the pixel detection plane disposition. An approach to the real emission distributions was made and results show that irradiating the full surface of the four CIPHER modules does not change the polarimetric properties. For a $100 \%$ polarised Crab nebula type emission a $Q$ factor of 0.33 was calculated. The polarimetric performances did not show a significant degradation when typical balloon born experiment external factors are present, namely beam inclination and background noise. We showed that the beam inclination could give rise to important distortions of the $Q$ factor for beam inclinations greater than $5^{\circ}$ with respect to the optical axis of the instrument. This effect is amplified for energies higher than $300 \mathrm{keV}$. However, the CIPHER instrument predicted pointing accuracy of better than $1^{\circ}$ would guarantee that the polarimetric characteristics of the instruments would not be affected. The irradiation model combining the Crab polarised 
radiation flux with measured background noise rates showed that the CIPHER instrument polarimetric performance is only slightly affected when background was reduced through the use of a full active shielding system. Furthermore, the CIPHER telescopes minimum detectable polarisation is lower than $20 \%$ for observation times of $10^{3} \mathrm{~s}$ and lower than $1 \%$ for $10^{6} \mathrm{~s}$ (satellite observation times), when measuring Crab nebula emission in the presence of a typical background noise rate obtained on board a balloon borne experiment. With this performance, the CIPHER instrument will be able to measure the polarisation of the emission from the most of polarised astrophysical sources. Although CIPHER Monte Carlo model results were obtained considering an ideal detector and electronics response, herein important notions for future eventual adjustments and geometric optimisation of CIPHER polarimetric prototype were acquired.

\section{Acknowledgements}

This work was carried out in Departamento de Física da Universidade de Coimbra, Portugal, Laboratoire PHASE, CNRS, Strasbourg, France and IASF-Sezione di Bologna, CNR, Italy. One of the authors (R. M. Curado da Silva) was supported by the Fundação para a Ciência e Tecnologia, Portugal, through the research grant SFRH/BPD/11670/2002.

\section{References}

Aprile, E., Bolotnikv, A., Chen, D., Mukherjee, R. and Xu, F.: 1994, Astrophys. J. Suppl. 92, 689.

Caroli, E., Bertuccio, G., Cola, A., Curado da Silva, R. M., Donati, A., Dusi, W., Landini, G., Siffert, P., Sampietro, M. and Stephen, J. B.: 2000, 'Hard X-ray polarimetry with a thick CdTe position sensitive spectrometer', SPIE Proceedings on X-ray and Gamma-ray instrumentation for Astronomy XI, Vol. 4140, pp. 573-583.

Caroli, E., Stephen, J. B., Dusi, W., Bird, A. J., Dean, A. J., Bertuccio, G., Sampietro, M., Yu, W., Zhang, C., Curado da Silva, R. M., Siffert, P. and Reglero, V.: 2000, 'The CIPHER telescope for hard X and soft gamma-ray polarimetry', in M. L. McConnell and J. M. Ryan (eds), Proceeding Series of American Institute of Physics on The Fift Compton Symposium, AIP Conference Proceeding, Vol. 510, pp. 809-813.

Curado da Silva, R. M., Caroli, E., Stephen, J. B. and Siffert, P.: 2002, Proc. SPIE 4497 70-78.

Gehrels, N. and Winkler, C.: 1996, SPIE Proc. 2806, 210-216.

Kroeger, R. A., Johnson, W. N., Kinzer, R. L., Kurfess, J. D., Inderhees, S. E., Philips, B. F. and Graham, B.: 1996, 'Gamma ray instrument for polarimetry, spectroscopy, and imaging (GIPSI), SPIE Proceedings on Gamma-Ray and Cosmic-Ray Detectors, Techniques, and Missions, Vol. 2806, pp. 52-63.

Lei, F., Dean, A. J. and Hills, G. L.: 1997, Space Sci. Rev. 82, 309-388.

Matt, G., Feroci, M., Rapisarda, M. and Costa E.: 1996, Radiat. Phys. Chem. 48, pp. 403-411.

Namito, Y., Ban, S. and Hirayama: 1993, Nucl. Inst. Meth., A 332, 277-283.

Parsons, A. M., Stahle, C. M., Lisse, C. M., Babu, S., Gehrels, N. A., Teegarden, B. J. and Shu, P. K.: 1994, 'Room temperature semiconductor detectors for hard X-ray astrophysics', SPIE Proceedings on Gamma-ray Detector Physics and Application, Vol. 2305, pp. 121-132. 
Schönfelder, V. et al.: 1993, Astrophys. J. Suppl. 86, 657.

Slavis, K. R., Dowkontt, P., Duttweiler, F., Epstein, J., Hink, P., Huszar, G., Kalemci, E., Leblanc, P., Matteson, J., Pelling, M. R., Rothschild, R. E., Stephan, E., Tumer, T. and Visser, G.: 2000, 'Performance of a prototype CdZnTe detector module for hard X-ray astrophysics', SPIE Proceedings on X-Ray and Gamma-Ray Instrumentation for Astronomy XI, Vol. 4140, pp. 249-256.

Stephen, J. B., Caroli, E., Curado da Silva, R. M. and Foschini, L.: 2001, 'Gamma-ray polarisation measurements with INTEGRAL/IBIS', AIP Conference Proceedings series, presented in GammaRay Astrophysics 2001, Baltimore, Maryland, U.S.A.

Swinyard, B. M., Didcot C., Malaguti, G., Caroli, E., Dean, A. J. and Di Coco, G.: 1991, Proc. SPIE 1548, 94-105. 